\title{
Dermatophytosis in Small Animals
}

\author{
Antonella S. Mattei ${ }^{1 *}$, Márcia A. Beber ${ }^{2}$ and Isabel M. Madrid ${ }^{3}$ \\ ${ }^{1}$ University Federal of Rio Grande (FURG), Medical School, RS, Brazil \\ ${ }^{2}$ Undergraduate Student in Dentistry, Federal University of Pelotas (UFPel), Pelotas, RS, Brazil \\ ${ }^{3}$ Facuty of Veterinary, Zoonosis Center Control, Federal University of Pelotas (UFPel), Pelotas, RS, Brazil
}

Received: March 06, 2014; Accepted: September 19, 2014; Published: November 04, 2014

*Corresponding author: Antonella Souza Mattei, University Federal of Rio Grande (FURG), Medical School, RS, Brazil, Tel: +55(53)-91447884; E-mail: antonella.mattei@hotmail.com

\begin{abstract}
The purpose of this article was to briefly review about dermatophytosis in small animals. In the last years, the pet population has increased as well the interest in having animals as pets. Because of this, these small animals are more and more inserted in our daily life. The understanding of ringworm epidemiology, diagnosis, treatment and control in pets is very important for the reason that pets are often asymptomatic carriers of dermatophytes being important sources of infection and/or carriers of infection. So, it is very important to know about this disease to reduce the spread of zoophilic fungal infections in humans, considering the close contact between pets and their owners, especially between children and their pets.
\end{abstract}

Keywords: Cat; Dog; Exotic pet mammals; Microsporum spp.; Trichophyton spp.; Zoonosis; Dermatophytosis; Mycosis

\section{Abbreviations}

FeLV: Feline Leukemia Virus; FIV: Feline Immunodeficiency Virus; KOH: Potassium Hydroxide; Min: Minutes

\section{Introduction}

In recent years, both the number of domestic pets fed inside their owners' homes and the interest in having animals as pets at one's home have increased markedly. Considering the close contact between pets and their owners, especially between children and cats and dogs, these animals that are often asymptomatic carriers of dermatophytes can be important sources of infection and/or carriers of infection [1].

However, nowadays not only cats and dogs are popular pets, the exotic companion mammals (e.g. rabbits, rodents, ferrets, hedge hogs) are also common and they can be a potential source of zoonotic infections transmissible to their owners [2].

Dermatophytosis is characterized by a superficial skin infection confined to keratinised epithelium, is the most common fungal disease in clinical veterinary medicine in small animals. These fungi produce keratinases and others enzymes capable to digest the keratin protein complex, allowing the dermatophyte to burrow deeper into the stratum corneum in the host and therefore to elicit an inflammatory reaction. The degree of inflammation, dependent of host-fungus interaction, determines the degree and significance of the clinical signs $[3,4]$.
Originally soil inhabitants, dermatophytes have evolved to infect animals and humans and are accordingly classified into geophilic, zoophilic, and anthropophilic species based on their main habitat or host. Three dermatophytes genera Trichophyton, Microsporum, and Epidermophyton-comprise more than 40 different species $[4,5]$.

Animals can be infected by a great variety of dermatophytes, mostly zoophilic but also geophilic species, and exceptionally anthropophilic dermatophytes (Table 1) [6].

Clinical importance is usually mild except in heavily infected young animals such as calves with an impact on general health condition and growth. On the contrary, serious economic consequences result from the long duration of the disease $[1,6]$.

The understanding of ringworm epidemiology, diagnosis, treatment and control in pets is very important for reducing the spread of fungal infections in animals and humans. The purpose of this article was to briefly review about dermatophytosis in small animals.

Table 1: Zoophilic and Geophilic dermatophytes species in animals.

\begin{tabular}{|l|c|c|}
\hline Dermatophytes species & Main source & Others \\
\hline Microsporum canis & Cat, dog, horse & All mammals \\
\hline Microsporum gallinae & poultry & Dog, cat \\
\hline Microsporum gypseum & Soil & All mammals \\
\hline Microsporumn nanum & Soil & Pig \\
\hline Microsporum persicolor & Microtid rodents & Dog, cat \\
\hline Trichophyton equinum & Horse & Cat, dog (rare) \\
\hline Trichophyton erinacei & Hedgehog & Dog \\
\hline $\begin{array}{l}\text { Trichophyton } \\
\text { mentagrophytes }\end{array}$ & Rodents & All mammals \\
\hline Trichophyton simii & Primate & Fowl, dog, cat \\
\hline Trichophyton verrucosum & $\begin{array}{c}\text { Cattle, other } \\
\text { ruminants }\end{array}$ & All mammals \\
\hline
\end{tabular}

Note: Other geophilic species are frequently isolated from hair coat of mammals, but, usually, without pathogenic significance in animals: $M$. cookei, M. praecox (frequent in the equine environment and sometimes pathogenic for man), T. ajelloi, T. terrestre. Some rare cases of animal ringworm due to anthropophilic dermatophytes (E. floccosum, T. rubrum and T. tonsurans in dog; T. violaceum in pig) have been reported. 


\section{Etiology}

The etiologic agents of the dermatophytoses are classified in three genera, Epidermophyton, Microsporum, and Trichophyton $[4,7]$. The descriptions of the genera are essentially followed by the colony macroscopic aspects (diameter, texture, surface color and reverse color) and themicroscopic aspects (conidial morphology and formation of conidia). They both are updated following the discovery of new species. The genera and their descriptions are as follows:

Epidermophyton spp: The type species is Epidermophyton floccosum (Figure 1A and B). The colony aspects are filamentous turning into powder surface, central poor radial grooves and white color turning into yellow-green or mustard-yellow and brown-yellow reverse in Sabouraud dextrose agar at $25^{\circ} \mathrm{C}$. The macroconidia are broadly clavate with typically smooth, thin to moderately thick walls and one to nine septa, 20 to 60 by 4 to $13 \mathrm{~mm}$ in size. They are usually abundant and borne singly or in clusters. Microconidia are absent. This genus has only two known species to date, and only E. floccosum is pathogenic [7-11].

Microsporum spp: The type species is Microsporum canis (Figure 2A and B). The colony aspects are plane, velvety or cottony surface, white or yellowish color and brown or goldenyellow reverse in Sabouraud dextrose agar at $25^{\circ} \mathrm{C}$. Macroconidia are characterized by the presence of rough walls which may be asperulate, echinulate, or verrucose. They are fusiform, obovate (egg shaped) or cylindrofusiform. They may also have thin, moderately thick to thick walls and 1 to 15 septa and range in size from 6 to 160 by 6 to $25 \mathrm{~mm}$. Microconidia are sessile or stalked and clavate and usually arranged singly along the hyphae or in racemes [7-11].

Trichophyton spp: The type species is Trichophyton mentagrophytes var. Mentagrophytes (Figure 3A and B). The colony aspects are plane, white to cream color, powdery to granular surface and reverse yellowish brown to reddish-brown in Sabouraud dextrose agar at $25^{\circ} \mathrm{C}$. Macroconidia, when present, have smooth, usually thin walls and one to 12 septa, are borne singly or in clusters, and may be elongate and pencil shaped clavate, fusiform, or cylindrical. They range in size from 8 to 86 by 4 to $14 \mathrm{~mm}$. Microconidia, usually more abundant than macroconidia, may be globose, pyriform or clavate, or sessile or stalked, and are borne singly along the sides of the hyphae or in grape-like clusters [7-11].

In 1999, the Trichophyton tonsurans, T. mentagrophytes and related species and varieties were reclassified into 5 taxa, T. tonsurans, T. interdigitale (anamorph of Arthoderma vanbreuseghemii), T. mentagrophytes, T. simii (anamorph of $A$. simii) and T. erinacei (anamorph of A. benhamiae). In 2007, this was chaged by treating T. mentagrophytes as the anamorph of $A$. simii in place of T. simii and adding another anamorph of A. benhamiae, Trichophyton spp. Furthermore, in 2010 T. mentagrophytes was replaced by $T$. simii and treated again as an individual species, i.e. T. mentagrophytes var. quinckeanum [12].

Nowadays, the three genera, Epidermophyton, Microsporum, and Trichophyton, can be identified based on molecular taxonomic technique [13].
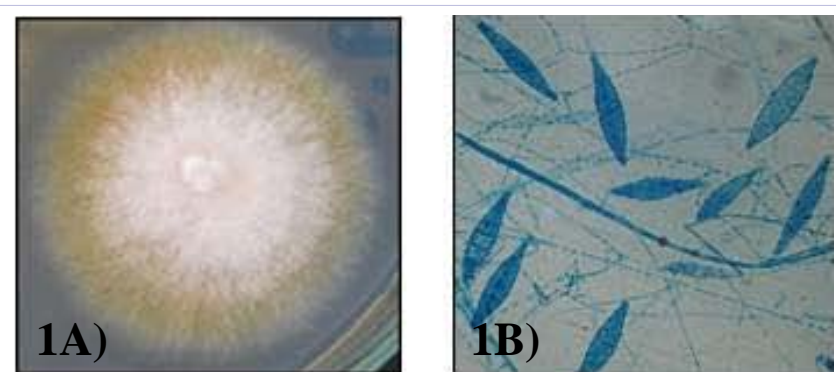

Figure 1: Microsporum canis culture, macroscopic colony (A) and microscopic observation in lactophenol (B) (Veterinary Mycology Laboratory, MICVET, Federal University of Pelotas, Rio Grande do Sul, Brazil).
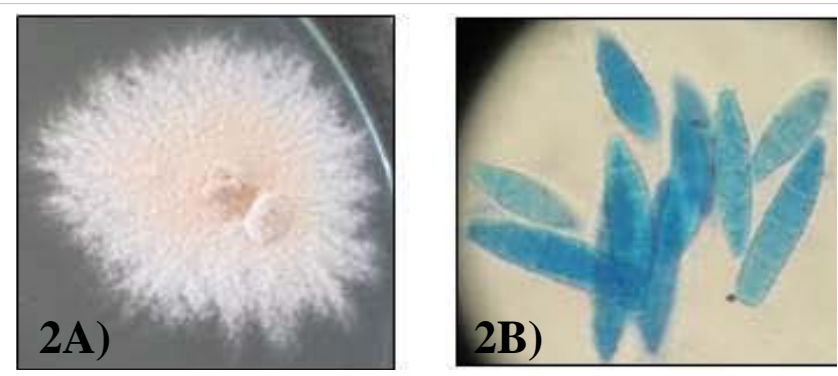

Figure 2: Microsporum gypseum culture, macroscopic colony (A) and microscopic observation in lactophenol (B) (Veterinary Mycology Laboratory, MICVET, Federal University of Pelotas, Rio Grande do Sul, Brazil).
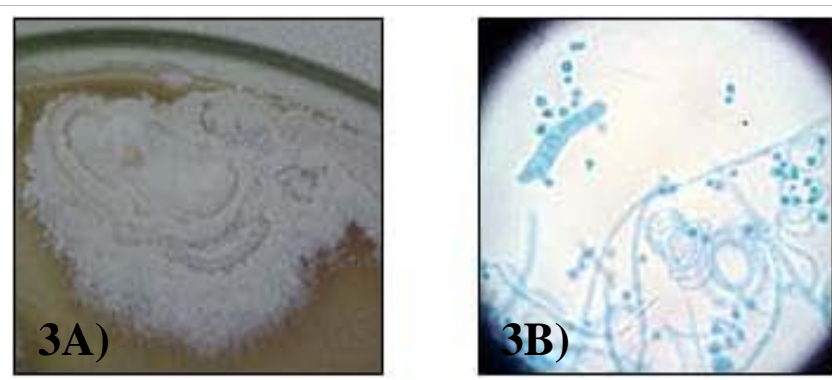

Figure 3: Trichophyton mentagrophytes var. mentagrophytes culture, macroscopic colony (A) and microscopic observation in lactophenol (B) (Veterinary Mycology Laboratory, MICVET, Federal University of Pelotas, Rio Grande do Sul, Brazil).

There are genotypic and phenotypic variations between isolates of the same species of dermatophyte. The clinical application of these variations has to be discovered yet. These variations do not reflect differences in symptomatology or epidemiological data [14].

The fungal pathogenicity factors, such as enzyme production, were associated with different clinical dermatophytosis pictures. Apparently, studies examining the enzyme production through molecular characterization and expression during infection are an importante means to understand the details of pathogenicity and may also answer questions related to the different degrees of virulence observed in different hosts [14-17].

On the basis of dermatophyte primary habitat associations, they are separated into three species: anthropophilic, 
zoophilic, and geophilic. Anthropophilic dermatophytes are main related with humans and unusually infect other animals (e.g. Epidermophyton floccosum). Zoophilic dermatophytes commonly infect animals or are associated with animals however sometimes can infect humans (e.g. Microsporum canis). Geophilic dermatophytes are found on soil together with keratinous human or animal materials (hair, feathers and scales) (e.g. Microsporum gypseum). These species may cause infection in human and animals [4, 6-10,18].

Microsporum canis, along with M. gypseum and T. mentagrophytes var. mentagrophytes, are the fungal species responsible for more than $95 \%$ of all dermatophytosis cases in pets $[3,18]$.

Carriage of $M$. canis is quite frequent in cats. The animal can be asymptomatic though some infected hairs are present. This mechanical carriage, not associated with infection, will be revealed through fungal cultures $[6,19,20]$. Whereas, the exotic pet mammals can be asymptomatic carriers of T. mentagrophytes var. mentagrophytes [2].

\section{Epidemiology}

A large variety of species have been isolated from domestic, captive and free-living animals, but some of them are more often associated with a particular host. The situation is more diversified with the geophilic dermatophytes that multiply in soil as saprobes and can become parasites for some of them $[6,21]$. The prevalence of dermatophytoses varies according to climate, temperature, relative humidity and precipitation in different geographical regions, as well as the natural reservoirs [20].

In Italy, M. canis is the most common dermatophyte isolated from dogs and from tinea capitis and tinea corporis in men. Geophilic dermatophytes species (M. gypseum and T. terrestre) have been isolated in dogs with a prevalence ranging from 2.2 to $8.7 \%$ [22]. This variation allegedly occurs due to difference in temperature, climate, relative humidity and precipitation among the geographical regions where the surveys were executed, along with the degree of experience of clinicians collecting the samples $[20,22]$.

The prevalence of dermatophytes in dogs range from $4 \%$ to $10 \%$ and a few studies show a higher prevalence $[3,20]$. However, in cats, the frequency of positive findings is greater than $20 \%$. These animals can be asymptomatic, with higher percentage and carriers of dermatophytes, important sources of infection and/or carriers of infection [20].Canine dermatoses were investigated in Brazil during a 1998-2003 period, and 107 (9.8\%) out of 1,089 dogs had dermatophytes represented by $M$. canis for $6.6 \%$ of the animals and M. gypseum for 3.0\% [6].

In the frequency study about exotic pet mammals, Dermatophytosis was present in 50\% (23/46) guinea pigs, $32.6 \%(15 / 46)$ in rabbits and $8.7 \%(4 / 46)$ in chinchillas. In all cases, T. mentagrophytes was the only dermatophyte identified. The remaining $8.7 \%$ were composed of ferrets [6.5\% (3/46)], in which both Microsporum spp. (2/3) and T. mentagrophytes $(1 / 3)$ were identified. And 2.2\% (1/46) were rats affected by dermatophytosis due to Microsporum spp. The most common dermatophyte identified was T. mentagrophytes (53.1\%), whereas Microsporum spp. occurred only in 3.7\% of the cases [2].

In Brazil, the study about the occurrence of yeasts and dermatophytes in shearing tools used in pet shops as well as veterinary offices and clinics, the fungal isolation was positive in 58 samples $(\mathrm{n}=150), 58.6 \%$ of which from clippers and $41.4 \%$ from slickers, resulting in 78 yeast isolates; but, filamentous fungi from the dermatophytes group were not found [23]. The epidemiological data regarding distribution of dermatophytes in veterinary environments are scare.

Transmission of dermatophytic infections between conidia from the environment or from existing hosts and new recipients can occur through direct contact with infected animals or indirectly from contaminated fomites, all circumstances that favour those contacts act as predisposing factors. Conidia are very resistant and may remain viable in the environment for years $[4,6]$, this explains why the use of material that is shared between animals favours the contamination. For geophilic dermatophytes, the soil is the reservoir in which the fungi multiply. Thus, the risk of contamination is higher for animals with outdoor contacts $[6,20,22]$.

Maraki and Tselentis [24] analyzed 111 cases of human dermatophytosis due to $M$. canis according to the origin of infection; in 15 cases (13.5\%) the infection was transmitted by humans, in 91 cases (82\%) the infection transmitted by cats and in only five cases $(4.5 \%)$ was the infection was transmitted by dogs. Katoh et al. [25] examined the scalps of patients with dermatophytosis due to $M$. canis yet without scalp lesions and the scalps of their family members without dermatophytoses. Dermatophyte fungi were detected in $93.8 \%$ of the scalps of those who lived in homes where cats were kept and in only $25 \%$ of homes without cats.

Animals younger than one year-old appear to be susceptible to dermatophytoses, but there is no apparent sex predisposition [2,5,26-28].

As for breed, Yorkshire terriers had a statistically significant higher prevalence of dermatophytoses, mainly caused by M. canis (46.4\%), than other breeds. This could be explained by qualitative and/or quantitative differences in non-specific cutaneous defences, sebum and sweat, rather than by their long hair. Geophilic dermatophytic species, instead, were mainly found on sheepherders and half-breed dogs probably due to different life style factors rather than to breed. Persian cats were infected only by M. canis while European and half-breeds were also affected by geophilic dermatophyte species. The high prevalence of infection by $M$. canis in Persian cats may be explained by the fact that they are more likely to be kept together in large groups where $M$. canis may readily spread. The presence of Geophilic dermatophyte species in European and half-breed cats can be explained by their specific life style, namely their tendency to live in the outdoor environment in contact with the soil [22].

Mere exposure to dermatophyte spores does not guarantee infection. An unknown but "critical mass" of spores must come 
into contact with a susceptible host. The spores must evade host defence mechanisms that include mechanical removal, competition with normal bacterial and fungal flora, exposure to fungistatic properties of epidermal lipids, low humidity of the skin surface and acquired host immunity [29].

Factors that favour infection include any pre-existing disease that will cause an increase in surface humidity, cause microtrauma to the skin and/or compromise host immune surveillance. Once a nidus of infection has been established, the fungal species proceeds to invade the keratin of hairs and skin and establish infection. Recovery from infection is dependent upon a competent cell mediated immune response [4,29].

The concomitance of diseases such as hyperadrenocorticism, or the use of some treatments, mainly corticotherapy, may favour appearance and worsening of fungal lesions through impairment of immunity. In cats, the Feline Immunodeficiency Virus (FIV) and the Feline Leukemia Virus (FeLV) are the potential immunosuppressive effect for developed this mycosis [6,18].

\section{Clinical Presentation}

The general presentation of ringworm in animals is a regular and circular alopecia, with erythematous margin and a thin desquamation. Pruritus is generally absent although described in a noticeable proportion of animals in some surveys. Lesions can be single or multiple, and are localized on any part of the animal although the anterior part of the body and the head seem more frequently involved. Usually, there is a centrifugal spread of lesions. Multiple lesions may coalesce, while a spontaneous healing at the center with regrowth of hairs is generally observed $[6,18]$.

Kerion (nodular dermatophytosis) is a round and nodular edematous reaction to dermatophyte infection, with a patch of erythematous scaling alopecia, characterized by a granulomatous dermatitis to a deep pyogranulomatous inflammation. In animals it is caused mainly by the species of dermatophytes M. gypseum, M. canis and T. mentagrophytes [18,30,31]. Ferreira et al. [32] described a kerion by M. gypseum causing a circular lesion on the nostril in a Dachshund, the first case report in Brazil.

Mycetoma-like lesions have been reported, mostly in Persian cats, in which the dermatophyte develops in the dermis and the subcutis. At examination, single or multiple cutaneous nodules, firm and not painful at palpation, usually situated on the back, the neck and tail basis are visible. Skin on the nodule is darkened with a blue or violaceous color, without alopecia or erythema. Fistulisation of the nodule is possible $[6,30,31,33]$.

\section{Diagnosis}

Wood's lamp examination looks for fluorescence on the hair shafts of $M$. canis-infected hairs. Infections by M.canis, approximately $50 \%$ fluoresence, besides this, several flaws in this technique, the use and interpretation. This examination is a screening tool and it is helpful for identification of hairs for direct examination and/or culture; a negative test does not rule out infection [18].

Direct examination of hairs and scales looks for the presence of fungal hyphae and/or ectothrix spores. This procedure can be done with clearing agents such as Potassium Hydroxide $(\mathrm{KOH})$ 10 or $20 \%[9,10,34]$. The positivity values for the $\mathrm{KOH}$ direct test were considered predictive in relation to positive cultures, which are considered as the gold standard. Fungal elements were observed in $61.0 \%$ of the cases analyzed. The sensitivity of this test varied from one fungus to another, with the clinical specimen examined originating from the more sensitive dogs making up a total of $63 \%$, and in cats $57.1 \%$. There were no false positives, but false negatives were seen, $5.8 \%$ in dogs and $20 \%$ in cats. Thus, it is possible to conclude that direct examinations could be very important in the diagnosis of dermatophytoses, since, if carried out by experienced professionals, it can certainly aid veterinarians by shortening the time required to initiate adequate treatment [29].

Definitive diagnosis is made by fungal culture. Fungal culture is considered the "gold standard" for diagnosis. The two most commonly used fungal culture media include Sabouraud's dextrose agar and dermatophyte test medium [9,10,34]. Culture is a valuable adjunct to direct microscopy and is essential at least in all nail infections and in any infection to be treated by systemic medication. In all cases, a medium selective against most nondermatophytic molds and bacteria is used as a primary isolation medium. Cycloheximide is incorporated into this medium as a semiselective agent to reduce the growth of nondermatophytic fungi. Sabouraud agar amended with cycloheximide and chloramphenicol is commonly used. Dermatophytoses recently treated with antifungal agents may repeatedly show uncultivable filaments and grow spurious molds, usually with revealing inconsistency from specimen to specimen [8-10,34].

Biochemical test were employed to differentiate Trichophyton spp. are enriched medias with urea, thiamine, histidine, nicotinic acid and inositol, the isolates are subcultured in this media [11]. Bromocresol purple (BCP)-casein-yeast extract agar for the rapid identification of T. verrucosum in specimens from rural areas, this medium turns from its sky blue original colour to violet within 4-7 days in the presence of T. mentagrophytes, thus permitting the differentiation with T. rubrum and M. persicolor. In addition, it contains casein which is hydrolysed by $T$. verrucosum and Trichophyton violaceum var. glabrum. When the isolate secretes proteases, a broad zone of clearing is observed in the opaque casein, surrounding the small colonies. The urea agar medium may therefore be useful to differentiate T. rubrum (urease -) from T. mentagrophytes var. interdigitale (urease +). With ureaseproducing strains, urea is splitted into ammonia, resulting in a $\mathrm{pH}$ change to alkaline and therefore in the change of the colour of the phenol redcontaining medium from a straw yellow to pink fuchsia after 2 (urea-indole broth) or 6 (Christensen's agar) days of incubation at $27^{\circ} \mathrm{C}$. Thus, T. verrucosum and T. concentricum require for growth the presence of thiamine and inositol. When the vitamin requirement is partial, a reduced growth is observed on control medium and for instance, thiamine stimulates Trichophyton tonsurans and T. violaceum. Nutritional test media are commercially available (Trichophyton agars $1-7$, Difco ${ }^{\circledR}$ ). Assimilation of sorbitol can also be used to differentiate T. rubrum 
(positive) from T. mentagrophytes (negative) [35].

Skin biopsy is helpful in the diagnosis of kerion reactions and granulomatous infections because cultures are often negative, but don't know the species involved. With the advent of molecular technology, it has been possible to compare the molecular and conventional taxonomy of dermatophyte species [29,34].

This diagnosis are based on the molecular taxonomic technique to identify and compare the isolate species for epidemiology studies [13].

\section{Treatment}

The therapeutic measures should include the combination of systemic and topical treatment. An appropriate duration of treatment must be respected [18].

Topical treatment with enilconazole, polyene and miconazole has consistent antifungal activity. The enilconazole should not be rinsed from the hair coat and a contact time of $10 \mathrm{~min}$ for miconazole shampoos is recommended for efficacy. Captan, chlorhexidine (as a single agent), and povidine iodine have been consistently ineffective antifungal agents when tested using isolated infective spores and/or hairs and are not recommended for use $[18,36]$. Topical therapy should be used in conjunction with systemic therapy; if cost constraints do not allow for concurrent systemic therapy, twice weekly applications of enilconazole can be used until a mycological cure (2-3 negative consecutive fungal cultures) are obtained [18].

Conventional systemic treatment relies on oral antifungal drugs such as, griseofulvin; itraconazole and terbinafine are all effective systematic antifungal agents. The combined systemic and topical treatment should be maintained for at least 10 weeks $[6,28]$.

Kerion lesions may require debridement or other local care, and the use of antibacterial agents may be indicated to treat secondary infections. Steroids such as prednisone may cause a significant decrease in inflammation [8]. Ferreira et al. [32] used a topical treatment for kerion in dog with miconazole, gentamicin and betametazone, twice a day. In 45 days, the lesion was completely recovered.

Lufenuron is a benzyl-urea phenol compound that inhibits chitin synthesis and is used as an insecticide. Its efficacy in the therapy of dermatophytosis in dogs and cats was evaluated in several clinical studies, with contradictory results. Zur and Elad [36] concluded that lufenuron had no inhibitory activity on dermatophytes in vitro or in vivo and its clinical use as an antifungal agent was questionable. An immunomodulatory effect of the drug was, however, possible.

\section{Prevention and Control}

Conidia are very resistant and may remain viable in the environment for years $[4,6]$. The major chance of infection occurs when contacted with infected animals or contaminated environments. So the best manner to avoid infection is to inhibit this contact. The prophylactic strategy would be very simple if these animals expressed obvious clinical signs [20,22,37].
There are studies reporting on the use of fungal vaccines in cats to prevent dermatophytosis. A killed dermatophyte vaccine for treatment of $M$. canis in cats is available (Fel-O-Vax, Fort Dodge Laboratories). This product is licensed and used for the treatment and prevention of lesions, but not diseases. In another study, an experiment was done using a combined live-inactivated dermatophyte vaccine and a killed commercially available dermatophyte vaccine were evaluated for prophylactic immunity and any therapeutic benefit. Neither vaccine prevented the infection nor provided a more rapid cure when compared with each other or with untreated control. However, vaccination was associated with a slightly reduced severity of initial infection when compared with controls. Interest in vaccination as a treatment or prophylaxis continues to be an area of intense research $[29,38]$.

\section{Conclusion}

Dermatophytoses are the most common infectious skin diseases detected in small animals, in veterinary clinics. The knowledge about epidemiology, clinical presentation and diagnosis of ringworm are important for treatment, prevention, and control, besides public health problem, as the pet population has increased in the last years and these animals are more inserted in our daily life, maintaining close contact with us, especially with our children. Moreover, we have to consider that fomites and the environment are the efficient sources of infection due to the high resistance of dermatophyte arthroconidia.

\section{Acknowledgement}

The authors thank Veterinary Mycology Laboratory (MICVET) of Federal University of Pelotas, Rio Grande do Sul, Brazil.

\section{References}

1. Ates A, Ilkit M, Ozdemir R, Ozcan K. Dermatophytes isolated from asymptomatic dogs in Adana, Turkey: A preliminary study. Journal of Medical Mycology. 2008; 18(3):154-157. DOI: 10.1016/j. mycmed.2008.07.001.

2. d'Ovidio D, Santoro D. Survey of Zoonotic Dermatoses in Client-Owned Exotic Pet Mammals in Southern Italy. Zoonoses and Public Health. 2014; doi:10.1111/zph.12100.

3. Copetti MV, Santurio JM, Cavalheiro AS, Boeck AA, Argenta JS, Aguiar LC. Dermatophytes isolated from dogs and cats suspected of dermatophytosis in Southern Brazil. Acta Scientiae Veterinariae. 2006; 34(2): 119-124.

4. Dahdah MJ, Scher RK. Dermatophytes. Current Fungal Infection Reports. 2008; 2: 81-86.

5. Coelho AC, Alegria N, Rodrigues J. Isolamento de dermatófitos em animais domésticos em Vila Real, Portugal. Arquivo Brasileiro de Medicina Veterinária e Zootecnia. 2008; 60(4)1017-1020. doi: 10.1590/S0102-09352008000400035.

6. Chermette R, Ferreiro L, Guillot J. Dermatophytoses in Animals. Mycopathologia. 2008; 166(5-6): 385-405. doi: 10.1007/s11046-0089102-7.

7. de Hoog GS. Atlas of Clinical Fungi, 2nd ed. Utrecht: Centraalbureau voor Schimmel cultures; 2000.

8. Weitzman I, Summerbell R. The Dermatophytes. Clinical Microbiology Reviews. 1995; 8(2): 240-259. 
9. Lacaz CS, Porto E, Martins JEC, Heins-Vaccari EM, Melo TN. Tratado de Micologia Médica, 9th ed. Prefácio: Bertrand Dupont. São Paulo: Sarvier, 2002. 44(5): 297-298.

10. Sidirm J. Micologia médica à luz de autores contemporâneos. Rio de Janeiro: Guanabara Koogan. 2004.

11. Matsumoto T, Ajello L. Current taxonomic concepts pertaining to the dermatophytes and related fungi. Int. J. Dermatol. 1987; 6(8): 491499.

12. Kawasaki M. Verification of a taxonomy of Dermatophytes based on mating results and phylogenetic analyses. Med Mycol J. 2011; 52(4): 291-295.

13. Grumbt M, Monod M, Staib P. Genetic advances in dermatophytes. FEMS Microbiol Lett. 2011; 320(2): 79-86. doi: 10.1111/j.15746968.2011.02276.x.

14. da Costa FV, Farias MR, Bier D, de Andrade CP, de Castro LA, da Silva SC, et al. Genetic variability in Microsporum canis isolated from cats, dogs and humans in Brazil. Mycoses. 2013; 56(5): 582-588. doi: 10.1111/ myc.12078.

15. Viani FC, Cazares Viani PR, Gutierrez Rivera IN, Gonçalves da Silva E, Rodrigues Paula C, Gambale W. Extracellular proteolytic activity and molecular analysis of Microsporum canis strains isolated from symptomatic and asymptomatic cats. Rev IberoamMicol. 2007; 24(1): 19-23.

16. White TC, Oliver BG, Graser Y, Henn MR. Generating and testing molecular hypotheses in the dermatophytes. Eukaryot Cell. 2008; 7(8): 1238-1245. doi: 10.1128/EC.00100-08.

17. Vermout S, Tabart J, Baldo A, Mathy A, Losson B, Mignon B. Pathogenesis of dermatophytosis. Mycopathologia. 2008; 166(5-6): 267-275.

18. Miller WH, Craig EG, Campbell KL, Muller GH, Scott DW. Muller \& Kirk's Small animal dermatology. 7th ed. St. Louis: Elsevier; 2013.

19. Nobre MO, Meireles MCA, Cordeiro JMC. Importância do felino doméstico na epidemiologia da dermatofitose por Microsporumcanis. Revista da Faculdade de Zootecnia, Veterinária e Agronomia. 2000/2001; 7/8(1): 81-84

20. Brilhante RS, Cavalcante CS, Soares-Junior FA, Cordeiro RA, Sidrim JJ, Rocha MFG. High rate of Microsporum canis feline and canine dermatophytoses in North east Brazil: Epidemiological and diagnostic features. Mycopathologia. 2003; 156(4): 303-308.

21. Costa M, Passos XS, Souza LK, Miranda AT, Lemos JA, Júnior JG, et al. Epidemiologia e etiologia das dermatofitoses em Goiânia, GO, Brasil. Revista da Sociedade Brasileira de Medicina Tropical. 2002; 35(1): 1922.

22. Cafarchia C, Romito D, Sasanelli M, Lia R, Capelli G, Otranto D. The epidemiology of canine and feline dermatophytoses in southern Italy. Mycoses. 2004; 47(11-12): 508-513.

23. Mattei AS, Madrid IM, Santin R, Schuch LFD, Meireles MCA. Occurrence of fungi with pathogenic potential in shearing tools. Archives of Veterinary Science. 2014; 19: 40-45.
24. Maraki S, Tselentis Y. Survey on the epidemiology of Microsporum canis infections in Crete, Greece over a five-year period. Int J Dermatol. 2000; 39(1): 21-24.

25. Katoh T, Sano T, Kagawa S. Isolation of dermatophyte from clinically normal scalps in $M$. canis infections using the hairbrush method. Mycopathologia. 1990; 112(1): 23-25.

26. Paixão GC, Sidrim JJC,Campos GMM, Brilhante RSN, Rocha MFG. Dermatophytes and saprobe fungi isolated from dogs and cats in the city of Fortaleza, Brazil. Arquivo Brasileiro de Medicina Veterinária e Zootecnia. 2001; 2001(5): 121-125. doi: 10.1590/S010209352001000500010 .

27. Mancianti F, Nardoni S, Cecchi S, Corazza M, Taccini F. Dermatophytes isolated from symptomatic dogs and cats in Tuscany, Italy during a 15-year-period. Mycopathologia. 2002; 156(1): 13-18.

28. Balda AC, Otsuka M, Larsson CE. Ensaio clínico da griseofulvina e da terbinafina na terapia das dermatofitoses em cães e gatos. Ciência Rural. 2007; 37(3): 750-754.

29. Moriello KA. Treatment of dermatophytosis in dogs and cats: review of published studies. Vet Dermatol. 2004; 15(2): 99-107.

30. Kano R, Edamura K, Yumikura H, Maruyama H, Asano K, Tanaka S, et al. Confirmed case of feline mycetoma due to Microsporum canis. Mycoses. 2008; 52(1): 80-83. doi: 10.1111/j.1439-0507.2008.01518.x.

31. Pereira AN, Damico CB, Souza HJ, Corgozinho KB, Graça R, Almeida EC, Ferreira AM. Pseudomicetoma dermatofítico causado por Microsporum canis em gato da raça persa. Acta ScientiaeVeterinariae. 2006; 34(2): 193-196.

32. Ferreira, Machado RR, da Silva ML, Spanamberg, Ferreiro A, Laerte . Quérion causado por Microsporum gypseumem um cão. Acta Scientiae Veterinariae. 2006; 34(2): 179-182.

33. Tostes RA, Giuffrida R. Pseudomicetoma dermatofítico em felinos. Ciência Rural. 2003; 33(2): 363-365.

34. Pérez J, Carrasco L. Diagnóstico histopatológico de micosisenpatología veterinária. Revista Iberoamericana de Micologia. 2000; 17: 18-22.

35. Robert R, Pihet M. Conventional Methods for the Diagnosis of Dermatophytosis. Mycopathologia. 2008; 166(5-6): 295-306. doi: 10.1007/s11046-008-9106-3.

36. Zur G, Elad D. In vitro and in vivo Effects of Lufenuron on Dermatophytes Isolated from Cases of Canine and Feline Dermatophytoses. J Vet Med B Infect Dis Vet Public Health. 2006; 53(3): 122-125.

37. Mignon B, Monod M. Zoonotic Infections with Dermatophyte Fungi. In: Palmer SR. Oxford Textbook of Zoonoses. New York: Oxford University Press; 2011.

38. Rybnikar A, Vrzal V, Chumela J, Petrás J. Immunization of cats against Microsporum canis. Acta Veterinary Brunensis. 1997; 66: 177-181. doi:10.2754/avb199766030177. 